\title{
Erratum to: CBLIB 2014: a benchmark library for conic mixed-integer and continuous optimization
}

\author{
Henrik A. Friberg1,2
}

Published online: 12 December 2015

(C) Springer-Verlag Berlin Heidelberg and The Mathematical Programming Society 2015

\section{Erratum to: Math. Prog. Comp. DOI 10.1007/s12532-015-0092-4}

The below statement was inadvertently missed out during the typesetting process. The correct text is provided below.

In Sect. 4.1, the list of certificates a solver could return for conic continuous optimization problems is not complete as claimed, as the paper was published without the following fifth point:

- certified dual facial reducibility when we are given a feasible point to problem (P), modified such that $b$ and $B$ are fixed to zero, with a zero-valued objective value, $c^{T} c+\langle C, X\rangle=0$ (within a tolerance), and non-zero entries of any self-dual cone. This is a facial reduction certificate for (D) showing it to be ill-posed in the sense of Renegar [2].

This certificate is needed when the problem $(\mathrm{P})$ has an unattained optimal solution or if the objective value can be improved indefinitely even though it has no improving ray (i.e., no certificate of dual infeasibility exists). Both cases are shown to occur in [1] where an algorithm is furthermore constructed with the property of always returning one of the five certificates. This shows the corrected list of certificates to be complete.

The online version of the original article can be found under doi:10.1007/s12532-015-0092-4.

\footnotetext{
$\bowtie$ Henrik A. Friberg

metronware@gmail.com

1 Department of Wind Energy, Technical University of Denmark, Roskilde, Denmark

2 MOSEK ApS, Copenhagen, Denmark
} 


\section{References}

1. Permenter, F., Friberg, H.A., Andersen, E.D.: Solving conic optimization problems via self-dual embedding and facial reduction: a unified approach. Technical report. http://www.optimization-online.org/ DB_HTML/2015/09/5104.html (2015)

2. Renegar, J.: Incorporating condition measures into the complexity theory of linear programming. SIAM J. Optim. 5(3), 506-524 (1995) 